Rend. Istit. Mat. Univ. Trieste

Vol. XXXVII, 1-38 (2005)

\title{
A Crash Course on Kleinian Groups
}

\author{
Caroline Series ${ }^{(*)}$
}

Summary. - These notes formed part of the ICTP summer school on Geometry and Topology of 3-manifolds in June 2006. Assuming only a minimal knowledge of hyperbolic geometry, the aim was to provide a rapid introduction to the modern picture of Kleinian groups. The subject has recently made dramatic progress with spectacular proofs of the Density Conjecture, the Ending Lamination Conjecture and the Tameness Conjecture. Between them, these three new theorems make possible a complete geometric classification of all hyperbolic 3-manifolds. The goal is to explain the background needed to appreciate the statements and significance of these remarkable results.

\section{Introduction}

A Kleinian group is a discrete group of isometries of hyperbolic 3space $\mathbb{H}^{3}$. Any hyperbolic 3-manifold is the quotient of $\mathbb{H}^{3}$ by a Kleinian group. In the 1960s, the school of Ahlfors and Bers studied Kleinian groups mainly analytically, in terms of their action on the Riemann sphere. Thurston revolutionised the subject in the 1970s by taking a more topological viewpoint and showing that in a certain sense 'many' 3-manifolds, perhaps one could say 'most', are hyperbolic. He also introduced many wonderful new concepts, some of which we shall meet here.

In the last five years, our understanding of Kleinian groups has advanced by leaps and bounds with the proofs of three great conjectures: the Density Conjecture, the Ending Lamination Conjecture

(*) Author's Address: Caroline Series, Mathematics Insitute, University of Warwick, Coventry CV4 7AL, England, email: cms@maths.warwick.ac.uk. 
and the Tameness Conjecture. Combined, they give a remarkably complete picture of Kleinian groups.

The aim of these notes is to give a rapid introduction to this vast subject. Our goal is to reach a point from which we can appreciate the statements and significance of the three conjectures. The first two chapters contain general background on the algebra, geometry and topology of Kleinian groups. Chapter 3 presents the classical and well understood picture of geometrically finite groups. In the last chapter, we describe spaces of groups and the Thurston-Bonahon picture of geometrically infinite ends.

There are only a few books which systematically take the modern viewpoint, notably [26, 19]. A new book by Marden [23], of which I was fortunate to have a preview, is due to appear shortly. It contains state of the art references and I am sure will become an essential handbook for anyone wanting to work on this subject seriously. These notes are essentially the same as those presented during the summer school with only minor editorial changes. I hope the informal style will prove user friendly. In particular, rather than referring to the original papers, I mainly give general references to books in which more detailed information can be found. A few topics, notably the Margulis lemma and the Mostow rigidity were covered in detail by other lecturers. I have added brief explanations to make these notes more self-contained. Time and space prevented the inclusion of other topics, in particular I should have liked to have a chapter on surfaces, Teichmüller theory and geodesic laminations. Fortunately this material is fairly easily available from various sources.

My title for these notes is not original. In 1974, Bers and Kra edited "A Crash Course on Kleinian groups" [4] which is still well worth consulting. Besides filling in much background omitted here, it is very striking that the recent progress has precisely resolved many questions already raised there.

I would like to thank the organisers of the meeting ICTP Summer School on Geometry and Topology of 3-manifolds for giving me the opportunity to present this beautiful material both by lecturing in the summer school and here in print. 


\section{Chapter 1: Kleinian group basics}

A Kleinian group is a discrete group of orientation preserving isometries of hyperbolic 3 -space $\mathbb{H}^{3}$. There are many reasons to study such groups. One important motivation is that they arise as the holonomy representation of a hyperbolic structure on a 3-manifold $M$. More precisely:

Theorem 1.1. Let $M$ be a complete hyperbolic 3-manifold. Then $M=\mathbb{H}^{3} / G$ where $G$ is a Kleinian group.

Here complete means that every geodesic can be extended indefinitely.

Since $M$ looks locally like hyperbolic space, $G$ contains no elements of finite order. So we will often find it convenient to simplify by assuming that $G$ is torsion free, in other words that there is no $g \in G$ with $g^{k}=$ id.

In this chapter we look at some basic properties of Kleinian groups. General references are $[2,23,26,33]$ and the much older but still useful book [17].

Modelling hyperbolic space We shall work with the upper half space and Poincaré ball models of hyperbolic 3 -space $\mathbb{H}^{3}$. This allows us to identify the orientation preserving isometry group $I s o m^{+} \mathbb{H}^{3}$ with the group $P S L(2, \mathbb{C})$.

The upper half space $\mathcal{H}$ consists of points $\{(z, t): z \in \mathbb{C}, t>0\}$. The metric is $d s^{2}=\frac{|d z|^{2}+d t^{2}}{t^{2}}$. In the unit ball $B^{3}=\left\{\left(x_{1}, x_{2}, x_{3}\right) \in\right.$ $\left.\mathbb{R}^{3}: r^{2}=x_{1}^{2}+x_{2}^{2}+x_{3}^{2}<1\right\}$ the metric is $d s^{2}=\frac{4\left|d r^{2}\right|}{\left(1-r^{2}\right)^{2}}$. Using stereographic projection (or rather its inverse) we can easily map $\mathcal{H}$ to $B^{3}$ so usually we consider these two models to be equivalent.

The model is conformal, that is, angles are correctly seen as Euclidean angles. In $\mathcal{H}$, hyperbolic planes are either vertical Euclidean planes or hemispheres centred on $\mathbb{C}$. Geodesics are vertical Euclidean lines or arcs of great circles on these hemispheres.

The hyperbolic distance $d_{\mathbb{H}^{3}}(P, Q)$ between $P=\left(z_{1}, t_{1}\right)$ and $Q=$ $\left(z_{2}, t_{2}\right)$ in $\mathcal{H}$ is given by

$$
\cosh d_{\mathbb{H}^{3}}(P, Q)=1+\frac{\left|z_{1}-z_{2}\right|^{2}+\left|t_{1}-t_{2}\right|^{2}}{2 t_{1} t_{2}} .
$$


In particular, we find

$$
d_{\mathbb{H}^{3}}\left(\left(0, t_{1}\right),\left(0, t_{2}\right)\right)=\left|\log \frac{t_{1}}{t_{2}}\right| .
$$

If $P \in \mathcal{H}$ is fixed, then $d_{\mathbb{H}^{3}}(P, Q) \rightarrow \infty$ as $t_{2} \rightarrow 0$. This justifies calling the Riemann sphere $\hat{\mathbb{C}}=\mathbb{C} \cup \infty$ the boundary at infinity. When the specific model is not important, we write this boundary as $\partial \mathbb{H}^{3}$.

Representation of isometries using $S L(2, \mathbb{C})$. As in Euclidean geometry, isometries of $\mathbb{H}^{3}$ are generated by reflections in (hyperbolic) planes. Orientation preserving isometries correspond to even numbers of reflections. Hyperbolic planes meet $\hat{\mathbb{C}}$ in circles and hyperbolic reflection in a plane extends to inversion in a circle. (Inversion in the circle $|z-c|=r$ is given by the formula $z \mapsto c+\frac{r^{2}}{z-c}$.) Thus $I$ som $^{+} \mathbb{H}^{3}$ is generated by products of even numbers of inversions. These are just the Möbius maps $z \mapsto \frac{a z+b}{c z+d}$. We briefly recall some basic facts on Möbius maps. There are many sources for details, for example $[17,31]$.

- Möbius maps compose by matrix multiplication. Multiplying all coefficients in $A=\left(\begin{array}{ll}a & b \\ c & d\end{array}\right)$ by a non-zero $\lambda \in \mathbb{C}$ does not affect the action. Hence dividing all coefficients by $\pm \sqrt{\operatorname{det} A}$, we may assume $A$ is normalised so that $\operatorname{det} A=1$. The ambiguity \pm 1 means that the group of Möbius maps Mob $\hat{\mathbb{C}}$ can be identified with $P S L(2, \mathbb{C})$.

- Mob $\hat{\mathbb{C}}$ acts transitively on triples of distinct points in $\hat{\mathbb{C}}$.

- Transforming $\hat{\mathbb{C}}$ by $S \in$ Mob $\hat{\mathbb{C}}$ induces the action of conjugation on $A \in \hat{\mathbb{C}}$. For example, if $A$ has a fixed point $z_{0}$ then $S A S^{-1}$ has a fixed point $S\left(z_{0}\right)$.

- $\operatorname{Tr} A=a+d$ is invariant under conjugation in $S L(2, \mathbb{C})$. Because of the ambiguity of sign, strictly speaking only $\operatorname{Tr}^{2} A$ is defined on $P S L(2, \mathbb{C})$. 
- A Möbius map has one or two fixed points. If there are two fixed points, one is attracting and one repelling. The classification up to conjugation is given below.

\section{Types of isometries}

One fixed point, Parabolic: $\operatorname{Tr} A= \pm 2$. Canonical form: $z \mapsto$ $z+c, c \in \mathbb{C}$.

Two fixed points $\operatorname{Tr} A \neq \pm 2$. Canonical form: $z \mapsto \kappa z, \kappa \in \mathbb{C}$. From the canonical form, $\operatorname{Tr} A=\kappa^{1 / 2}+\kappa^{-1 / 2}$. This subdivides into:

(Purely) hyperbolic: $\kappa>0 . \operatorname{Tr} A \in \mathbb{R}, \operatorname{Tr}^{2} A>4$.

Elliptic: $|\kappa|=1$. $\operatorname{Tr} A \in \mathbb{R}, \operatorname{Tr}^{2} A<4$.

Loxodromic: $\operatorname{Tr} A \notin \mathbb{R}$.

In $\mathbb{H}^{3}$, a parabolic $z \mapsto z+c$ extends to the Euclidean translation $(z, t) \mapsto(z+c, t)$. All the other types $z \mapsto \kappa z$ extend to a homothety $(z, t) \mapsto(\kappa z, \kappa t)$.

If $A \in \operatorname{Mob} \hat{\mathbb{C}}$ has two fixed points, then the line in $\mathbb{H}^{3}$ joining them is called its axis. By moving to the standard position with fixed points at $0, \infty$, it is easy to check that $A$ moves points along the axis by a distance $l_{A}$ and rotates around the axis by an angle $\theta_{A}$ given by

$$
\operatorname{Tr} A=2 \cosh \left(\left(l_{A}+i \theta_{A}\right) / 2\right) .
$$

(The ambiguity in sign caused by the fact that $\theta_{A}$ and $\theta_{A}+2 \pi i$ represent the same rotation, corresponds to the ambiguity in sign of $\operatorname{Tr} A$.) The positive real number $l_{A}$ is called the translation length of $A$ and $\lambda_{A}:=l_{A}+i \theta_{A}$ is called the complex translation length.

The map $A$ is purely hyperbolic iff $\theta_{A}=0$ and elliptic iff $l_{A}=0$. Note that an elliptic is just rotation about the axis, so has finite order iff $\theta_{A} \in 2 \pi \mathbb{Q}$. If $A$ is parabolic then by convention we set $l_{A}=\theta_{A}=0$.

Elementary groups The above discussion gives our first examples of Kleinian groups: 
1. $\left\langle g^{n}, n \in \mathbb{Z}\right\rangle, g$ parabolic.

2. $\left\langle g^{n} h^{m}, n, m \in \mathbb{Z}\right\rangle, g, h$ parabolic with same fixed point but different translation directions.

3. $\left\langle g^{n}, n \in \mathbb{Z}\right\rangle$, g loxodromic.

4. $\left\langle g^{n}, 0 \leq n<k\right\rangle, g$ elliptic, $g^{k}=\mathrm{id}$.

Actually these examples are not so special as they seem.

Lemma 1.2. Let $G$ be Kleinian and let $g, h \in G$. Then $g, h$ have either both fixed points in common or neither.

Proof. Normalise so that one common fixed point is at $\infty$ and then study $g^{-n} h g^{n}$. For example, if $h(z)=z+1$ and $g(z)=\kappa z$ with $\kappa>1$ then $g^{-n} h g^{n}(z)=z+\kappa^{-n}$. It follows easily that $G$ is not discrete.

(For a more detailed discussion of discreteness, see $\S 1.1$ below.) From this we deduce that if $g, h$ are loxodromics with a common fixed point, then they have the same axis.

Lemma 1.3. Let $G$ be Kleinian and let $g, h \in G$ be purely hyperbolic with the same axis. Then $G$ is cyclic.

Proof. Normalise such that $g(z)=\kappa z, h(z)=\mu z, \kappa, \mu>0$. The set of powers $\kappa^{n} \mu^{m}$ is discrete in $\mathbb{R}$ if and only if there exists $a>0$ such that $\kappa=a^{r}$ and $\mu=a^{s}$; moreover using the Euclidean algoritheorem we can assume that $a=\kappa^{p} \mu^{q}$ for suitable $p, q \in \mathbb{Z}$.

These and other similar results lead to:

Definition 1.4. A Kleinian group is elementary if it is virtually abelian; that is, it has an abelian subgroup of finite index.

Proposition 1.5. A Kleinian group is elementary iff there is a finite $G$-orbit for its action on $\hat{\mathbb{C}}$.

THEOREM 1.6. The list (1)-(3) above contains all the torsion free elementary Kleinian groups.

If a Kleinian group is not elementary, then it is quite complicated: 
Proposition 1.7. If a Kleinian group is not elementary then it contains infinitely many loxodromic elements with pairwise distinct axes.

In fact a group is non-elementary iff it contains a free subgroup on two generators, see proposition 2.30 below.

There are very few explicit tests to check if a group is discrete. Essentially the best we have is:

Theorem 1.8 (Jørgensen's inequality). Let $A, B \in S L(2, \mathbb{C})$. If $\langle A, B\rangle$ is Kleinian and non-elementary, then

$$
\left|\operatorname{Tr}^{2} A-4\right|+\left|\operatorname{Tr} A B A^{-1} B^{-1}-2\right| \geq 1 .
$$

As we shall see, this has many important implications.

Details of the above results can be found in many texts on Kleinian groups, for example [2, 17, 25, 23].

\subsection{More on discreteness}

Recall our definition: A subgroup $G \subset I s o m^{+} \mathbb{H}^{3}$ is Kleinian if it is discrete. With our identification of $I s o m^{+} \mathbb{H}^{3}$ with $S L(2, \mathbb{C})$, this is equivalent to: $G$ is Kleinian iff there exists $\epsilon>0$ such that $\|g \pm \mathrm{I}\|>$ $\epsilon$, where $\|$.$\| is any suitable norm on S L(2, \mathbb{C})$. This condition is enough to ensure that $G$ has no accumulation points, since $g_{n} \rightarrow g \in$ $G$ is equivalent to $g^{-1} g_{n} \rightarrow I$.

You may be worried about the $P S L(2, \mathbb{C})$ versus $S L(2, \mathbb{C})$. Here is a rather deep theorem:

TheOREm 1.9 ([14]). Let $\Gamma$ be an abstract group. Suppose given a representation $\rho: \Gamma \rightarrow P S L(2, \mathbb{C})$ such that $\rho(\Gamma)$ is Kleinian and non-elementary. Then $\rho$ lifts to $\hat{\rho}: \Gamma \rightarrow S L(2, \mathbb{C})$ iff $\Gamma$ contains no elements of order 2.

If $G$ acts on a topological space $X$, then in order to have a 'decent' quotient space $X / G$ we need to know that $G$ acts in a 'reasonable' way, such that, for example, the orbits form a Hausdorff space. This leads to

DeFinition 1.10. Suppose that $G$ acts by homeomorphisms on a topological space $X$. Then $H$ acts properly discontinuously if for all compact subsets $K \subset X,\{g \in G: g K \cap K \neq \emptyset\}$ is finite. 
Lemma 1.11. If $G \subset P S L(2, \mathbb{C})$ acts properly discontinuously on a topological space $X$, then $G$ is discrete.

Proof. If the result is false, then there exist $g_{n} \in G, g_{n} \rightarrow I$. Take $K$ to be a ball with compact closure. Then $g_{n} K \cap K \neq \emptyset$ for infinitely many $g_{n}$.

In general, the converse is false. For example, let $G=S L(2, \mathbb{Z}[i])$, the subgroup of $S L(2, \mathbb{C})$ whose entries are Gaussian integers $m+$ $i n, m, n \in \mathbb{Z}$. This is clearly discrete. However the $G$-orbits on $\hat{\mathbb{C}}$ 'pile up', as one can see by looking at the orbit of 0 under the subgroup $S L(2, \mathbb{Z})$. It is easy to see this consists of the extended rational numbers $\mathbb{Q} \cup \infty .{ }^{1}$ Nevertheless, we have the following crucial theorem:

Theorem 1.12. $G \subset P S L(2, \mathbb{C})$ is Kleinian iff it acts properly discontinuously on $\mathbb{H}^{3}$.

Proof. The main idea is that for any $r>0$, and $P=(0,1) \in \mathbb{H}^{3}$, $\left\{\gamma \in S L(2, \mathbb{C}): d_{\mathbb{H}^{3}}(P, \gamma P)<r\right\}$ is compact in $S L(2, \mathbb{C})$. For details see for example $[2,33]$.

Corollary 1.13. If $G \subset P S L(2, \mathbb{C})$ is Kleinian and torsion free then $\mathbb{H}^{3} / G$ is a hyperbolic 3-manifold.

There are lots of possible variations of the definition of proper discontinuity. There is an excellent discussion in [33] $§ 3.5$.

Action on $\hat{\mathbb{C}}$ What about the action of a Kleinian group on $\hat{\mathbb{C}}$ ? The above example of $S L(2, \mathbb{Z}[i])$ shows it may not be properly discontinuous.

Definition 1.14. The ordinary set $\Omega=\Omega(G) \subset \hat{\mathbb{C}}$ of a Kleinian group $G$ is the maximal open subset of $\hat{\mathbb{C}}$ on which $G$ acts properly discontinuously. The limit set $\Lambda=\Lambda(G)$ is $\hat{\mathbb{C}} \backslash \Omega$.

\footnotetext{
${ }^{1}$ Groups with entries in number fields form a large and important class of Kleinian groups which we shall not have time to touch on in these notes, see [21].
} 
The set $\Omega$ is also called the regular set and the domain of discontinuity. It is the maximal set on which the elements of $G$ form a normal family in the sense of complex analysis. McMullen calls $\Lambda$, 'the chaotic set'. It is the analogue of the Julia set for a rational map. Typically, $\Lambda$ is very complicated. For a wide variety of pictures of limit sets and instructions on how to generate them, see [31].

If $G$ consists entirely of elliptic elements (and hence is finite), $\Lambda$ is empty. Otherwise, $\Lambda$ is non-empty, because $\hat{\mathbb{C}}$ is compact and the (infinite) $G$-orbits have to accumulate somewhere. The regular set $\Omega$ may or may not be empty. In some rather old fashioned terminology, $G$ is called 'of the first kind' if $\Omega=\emptyset$ and 'of the second kind' otherwise. The group $S L(2, \mathbb{Z}[i])$ is a good example of a group of the first kind. The elementary groups are all of the second kind; in fact:

Lemma 1.15. $\Lambda$ is finite iff $G$ is elementary. In this case, $\Lambda$ contains either 0,1 or 2 points.

Suppose $G$ is non-elementary. Then:

1. $\Lambda$ is uncountably infinite, closed and minimal, that is, the $G$ orbit of any point in $\Lambda$ is dense in $\Lambda$.

2. $\Lambda$ is the closure of the loxodromic fixed points (and equally of the parabolic fixed points, if $G$ contains any parabolic).

3. $\Lambda$ is the set of accumulation points of the $G$-orbit of any point in $\hat{\mathbb{C}} \cup \mathbb{H}^{3}$.

4. Suppose that $H$ is a subgroup of $G$. If either $H$ is of finite index, or $H$ is normal in $G$, then $\Lambda(H)=\Lambda(G)$.

5. If $\Lambda(G) \neq \hat{\mathbb{C}}$, then its interior is empty.

The last item suggest Ahlfors' conjecture:

Conjecture 1.16. If $\Lambda(G) \neq \hat{\mathbb{C}}$, then it has Lebsgue measure 0.

A consequence of the three new theorems mentioned in the introduction, is that Ahlfors' conjecture is now proved [16]. More precisely, Ahlfors proved his conjecture for geometrically finite groups (see Chapter 3). Following on work of Thurston and Bonahon about 
geometrically infinite groups (see Chapter 4), Canary proved it for tame groups [11]. Thus the Ahlfors conjecture now follows from the tameness theorem 4.53 .

Here are some properties of $\Omega$. Assume that $G$ is finitely generated, non-elementary and that $\Omega \neq \emptyset$. Then:

1. $\Omega$ has either 1,2 or infinitely many connected components.

2. Each connected component of $\Omega$ is either simply connected or infinitely connected.

3. If there are (at least) two $G$-invariant components $\Omega_{1}$ and $\Omega_{2}$, then each is simply connected and $\Omega=\Omega_{1} \cup \Omega_{2}$.

4. If there is one $G$-invariant component $\Omega_{0}$, then all other components are simply connected.

property (1) is easy, (2) is not hard using the Ahlfors finiteness theorem below, while $(3,4)$ are most easily proved using 3dimensional topology, see $[22,23]$. The groups in (3) are exactly the quasifuchsian groups we shall meet in the next chapter. In the older literature, groups with an invariant component are called function groups. Groups with a simply connected invariant component are called B-groups.

The Ahlfors finiteness theorem. We end this chapter with two deep theorems. Recall that a Riemann surface is a surface with local charts to $\mathbb{C}$ such that the overlap maps are complex analytic. This is called a conformal structure on the surface. A puncture is a neighbourhood of a boundary component for which we have a chart to a punctured disk. If a surface carries a hyperbolic structure, it automatically carries a conformal structure by 'forgetting' the metric and remembering only the angles. A Riemann surface is said to have finite type if it has finite genus and finitely many punctures. One version of the famous uniformisation theorem states that:

Theorem 1.17 (Uniformisation theorem). Suppose that $S$ is a Riemann surface, possibly with punctures, which has negative Euler characteristic. Then there is a unique hyperbolic structure on $S$ inducing the given conformal structure. 
Theorem 1.18 (Ahlfors' finiteness Theorem). Suppose that the Kleinian group $G$ is non-elementary and finitely generated. Then $\Omega / G$ is a finite union of Riemann surfaces of finite type. Moreover each of these surfaces has negative Euler characteristic and so is hyperbolisable.

The surfaces in $\Omega / G$ are together called the conformal boundary of $\mathbb{H}^{3} / G$ at infinity. There are (at least) two modern proofs of the Ahlfors finiteness theorem which are much easier than Ahlfors' original version, see [19] and [24].

\section{Chapter 2: Geometry of hyperbolic 3-manifolds}

In this chapter we look at some basic features of a hyperbolic 3manifold $M=\mathbb{H}^{3} / G$. As we shall see, what we learnt in the last chapter about the action of $G$ on $\hat{\mathbb{C}}$ gets us quite a long way.

First, consider loops in $M=\mathbb{H}^{3} / G$. We can identify $G$ with $\pi_{1}(M)$. Take any homotopically non-trivial loop $\gamma \subset M$. Let $L([\gamma])=\inf \left\{l\left(\gamma^{\prime}\right): \gamma^{\prime} \in[\gamma]\right\}$, where $[\gamma]$ is the free homotopy class of $\gamma$. Then either

- $L([\gamma])>0$, in which case the infimum is attained by a unique closed geodesic in the homotopy class and $L([\gamma])$ is the translation length of the associated isometry $g \in G$, or

- $L([\gamma])=0$, in which case $\gamma$ is represented by a parabolic element in $G$.

From Theorem 1.6 and proposition 1.7 in the last chapter we obtain:

COROLLARY 2.19. Suppose that $G$ is a non-elementary Kleinian group. Then:

1. $\mathbb{H}^{3} / G$ contains infinitely many distinct closed geodesics.

2. Rank 2 abelian subgroups correspond bijectively to pairs of parabolics with a common fixed point.

As we shall see, (2) implies that $M$ is atoroidal. 
Incompressible and compressible boundary If $G$ is a Kleinian group, it is often convenient to add on the conformal boundary at infinity to obtain the Kleinian manifold $M_{G}=\left(\mathbb{H}^{3} \cup \Omega\right) / G$. In the last chapter we saw that each connected component $\Omega_{i}$ of $\Omega$ is either simply connected or multiply connected. This has a nice interpretation in terms of the topology of $M_{G}$.

Let $\Omega_{i}$ be a connected component of $\Omega$. There is a natural inclusion map from the surface $\Omega_{i} / G$ into $M_{G}$ which induces a map $\iota: \pi_{1}\left(\Omega_{i} / G\right) \rightarrow \pi_{1}(M)=G$.

Lemma 2.20. $\iota$ is injective iff $\Omega_{i}$ is simply connected.

Proof. First suppose that $\Omega_{i}$ is not simply connected. Then it contains a closed loop $\gamma$ which is not trivial in $\Omega_{i}$ and hence not trivial in $\pi_{1}\left(\Omega_{i} / G\right)$. However the 'roof' sitting over $\gamma$ in $\mathbb{H}^{3}$ provides a null homotopy of $\gamma$ in $\mathbb{H}^{3} \cup \Omega$ and hence in $M_{G}$. So $\iota$ is not injective.

Now suppose that $\iota$ is not injective. This means there is a loop on $\Omega_{i} / G$ which is non-trivial in $\pi_{1}\left(\Omega_{i} / G\right)$ but trivial in $G$. By the loop theorem ${ }^{2}$, there is a loop on $\Omega_{i} / G$ which bounds a disk in $M_{G}$. This disk lifts to a disk in $\mathbb{H}^{3}$ whose boundary meets $\Omega_{i}$ in a closed non-trivial loop in $\Omega_{i}$.

If $\iota$ is injective, the corresponding boundary component is called incompressible, otherwise it is compressible. Usually the incompressible case is easier to handle.

Thick-thin decomposition and the Margulis lemma It turns out that the parts of a hyperbolic manifold which are 'thin', in the sense that there are short closed homotopically non-trival loops, have especially simple structure. More precisely, the injectivity radius of a Riemannian manifold $M$ at a point $x$ is the supremum of $r>0$ such that the $r$-ball with centre $x$ is embedded. The $\epsilon$-thin part of $M$ is the set of those points at which the injectivity radius is at most $\epsilon$. The celebrated Margulis lemma, applied in the context hyperbolic 2- or 3-manifolds, states that there is a universal $\epsilon_{0}>0$ such that whenever $\epsilon<\epsilon_{0}$, the $\epsilon$-thin parts of $M$ are all either toroidal collars

\footnotetext{
${ }^{2}$ Dehn's lemma and its consequence the loop theorem are fundamental results in 3-dimensional topology. For a detailed statement, see for example [19, 23].
} 
about short geodesics or horoball neighbourhoods of cusps, see below. This is known as the thick-thin decomposition of $M$, see [26, 33, 23]. We will be especially interested in the case of cusps.

Geometry of Cusps Suppose that $G$ contains a parabolic element. We want to see what $M$ looks like in a neighbourhood of the parabolic fixed point, which necessarily lies on the boundary $\partial \mathbb{H}^{3}$. Since the parabolic is represented by arbitrarily short loops in $M$, we know that this neighbourhood is in the thin part of the manifold and hence, by the Margulis lemma, is either a cusp cylinder or a cusp torus.

We can get a more explicit picture of cusp neighbourhoods as follows. In the upper half space model, a ball tangent to $\partial \mathbb{H}^{3}=\hat{\mathbb{C}}$ at $\xi \in \hat{\mathbb{C}}$ is called a horoball based at $\xi$. In particular, if $\xi=\infty$, a horoball based at $\infty$ in $\mathcal{H}$ is a set $H_{s}=\{(z, t): t \geq s\}$ for some $s>0$. We sometimes call $s$ the height of the horoball. Conjugating, we see that a parabolic with fixed point at $\xi$ maps horoballs based at $\xi$ to themselves.

Let $P$ be a Kleinian group all of whose elements are parabolic with a common fixed point. As we saw in Chapter $1, P$ is isomorphic either to $\mathbb{Z}$ (rank 1 ) or $\mathbb{Z}^{2}$ (rank 2 ). Working in $\mathcal{H}$, we can conjugate so that the fixed point is at $\infty$ and scale so that $P$ has generators $A(z)=z+1$, and additionally, in the rank 2 case, $B(z)=z+b$ where $\operatorname{Im} b \neq 0$. With this normalisation it is not hard to see that, in the rank 1 case, $H_{s} / P$ is a solid infinite cylinder with missing core (a cusp cylinder) and, in the rank 2 case, a solid torus with missing core (a cusp torus). The hyperbolic distance from $\partial H_{s}$ to the core of the cusp cylinder or cusp torus is infinite. The cusp cylinder is also infinite in the direction parallel to the core curve. Travelling along $\partial H_{s}$ in either direction parallel to the core curve we limit on the boundary at infinity $\hat{\mathbb{C}}$.

Now suppose that $P$ is a subgroup in a larger Kleinian group $G$. We want to see that this is also the correct picture in $\mathbb{H}^{3} / G$. For this, we need to show that for some choice of $s, H_{s} / P=H_{s} / G$. This will be the case if we can find $s$ such that $H_{s}$ is precisely invariant under $(P, G)$, meaning that:

- $g\left(H_{s}\right)=H_{s}$ for all $g \in P$ and 
- $g\left(H_{s}\right) \cap H_{s}=\emptyset$ for all $g \in G \backslash P$.

That we can do this is the content of the Margulis lemma in the cusp case. Jørgensen's inequality 1.8 allows us to quantify $s$ explicity.

Theorem 2.21. Suppose that the Kleinian group $G$ contains a parabolic $A(z)=z+1$. Then the interior of $H_{1}$ is precisely invariant under the parablic subgroup $P$ which fixes $\infty$.

The proof uses isometric circles. Suppose that $g \in S L(2, \mathbb{C})$ does not fix $\infty$. The isometric circle $I_{g}$ of $g$ is the circle in $\widehat{\mathbb{C}}$ on which $\left|g^{\prime}(z)\right|=1$. If $g=(a z+b) /(c z+d)$ we compute that $g^{\prime}(z)=(c z+d)^{-2}$ and hence that $I_{g}$ is the circle centre $-d / c$ and radius $1 /|c|$.

Lemma 2.22. (Shimizu's lemma) Suppose that the non-elementary group $G$ contains the parabolic $A=\left(\begin{array}{ll}1 & 1 \\ 0 & 1\end{array}\right)$. Then if $B=\left(\begin{array}{ll}a & b \\ c & d\end{array}\right) \in$ $G$, we have $|c| \geq 1$.

Proof. Jørgensen's inequality says that

$$
\left|\operatorname{Tr}^{2} A-4\right|+\left|\operatorname{Tr} A B A^{-1} B^{-1}-2\right| \geq 1 .
$$

We compute $\operatorname{Tr} A B A^{-1} B^{-1}=2+c^{2}$ so $|c| \geq 1$.

Proof of Theorem 2.21. Shimizu's lemma implies that if

$$
A=\left(\begin{array}{ll}
1 & 1 \\
0 & 1
\end{array}\right) \in G
$$

then for any $g=\left(\begin{array}{ll}a & b \\ c & d\end{array}\right) \in G$ not fixing $\infty$, the isometric circle $I_{g}$ has radius at most 1 . Let $\hat{I}_{g}$ be the hemisphere in $\mathcal{H}$ sitting above $I_{g}$. It is easy to check that $g$ maps the region in $\mathcal{H}$ outside $\hat{I}_{g}$ to the region in $\mathcal{H}$ inside $\hat{I}_{g^{-1}}$. This implies that the interior of the closed horoball $H_{1}$ is precisely invariant under $(P, G)$.

Cusps on $\Omega / G$ How does a parabolic in $\mathbb{H}^{3} / G$ relate to a puncture on the conformal boundary $\Omega / G$ ? Recall from the uniformisation theorem 1.17 that each surface in $\Omega / G$ carries a hyperbolic metric inducing its Riemann surface structure. A more general question is, how does the hyperbolic metric on $\Omega / G$ relate to the hyperbolic metric on $\mathbb{H}^{3} / G$ ? 
Theorem 2.23 (Ahlfors' Lemma). Suppose that $\Omega_{0}$ is a simply connected component of $\Omega$. Let $\gamma$ be a geodesic on $\Omega_{0} / G$, represented by an element $g \in G$ whose hyperbolic length in the unique hyperbolic structure on $\Omega_{0} / G$ is $l_{\Omega_{0} / G}(\gamma)$. Then

$$
l_{\mathbb{H}^{3} / G}(g) \leq 2 l_{\Omega_{0} / G}(\gamma) .
$$

In particular, if $\gamma$ is parabolic on $\Omega_{0} / G$ (so that $l_{\Omega_{0} / G}(g)=0$ ), then $g$ is parabolic in $G$.

By the two dimensional analogue of the discussion above, the neighbourhood of a puncture on a hyperbolic surface looks like the quotient of a horoball neighbourhood of $\infty$ by a translation; in other words, a cusp annulus. (Notice that on a hyperbolic surface, there are no rank 2 parabolic subgroups, because no such a subgroup has an invariant disk in $\hat{\mathbb{C}}$.) Such a neighbourhood projects to a topological disk in $\Omega_{0}$ tangent to the parabolic fixed point. The hemisphere above this disk is also precisely invariant under $P$. This means that on the end of the cusp cylinder we can glue in a punctured annulus, a neighbourhood of the puncture on $\Omega_{0} / G$. If this can be done on both ends of the cylinder, we call the quotient a pairing tube. This happens, for example, if $G$ is Fuchsian or quasifuchsian.

Notice that the inequality in Ahlfors' lemma only goes one way. This means that we could have a loop in $\Omega_{0} / G$ which was not parabolic (so not a loop round a puncture) which was nevertheless repesented by a parabolic element in $G$. Such curves are called accidental parabolics on $\Omega_{0} / G$. Typically, a curve which is accidentally parabolic on one component of $\Omega / G$ will represent a cusp on another.

The Kleinian manifold Recall we defined the Kleinian manifold associated to $G$ as $M_{G}=\left(\mathbb{H}^{3} \cup \Omega\right) / G$. If $G$ has cusps, $M_{G}$ is not compact. It is usually easier to deal with a compact manifold, so it is often convenient to remove (open) solid cusp annuli and tori round the cusps to create a compact manifold $M_{G}^{0}$. Corollary 2.19 shows that $M_{G}^{0}$ is always atoroidal, that is, all the $\mathbb{Z}^{2}$ subgroups come from boundary tori. Because of the hyperbolic structure it is also irreducible, that is, every 2 sphere bounds a ball. Compare a version of Thurston's celebrated hyperbolisation theorem: 
TheOREm 2.24. (Thurston, see [19]) Suppose that a compact 3 manifold $V$ is irreducible and atoroidal and that $\partial V \neq \emptyset$. Then $V$ has a hyperbolic structure, that is, $V$ is homeomorphic to $M_{G}^{0}$ for some Kleinian group $G$.

In a more elaborate version of this theorem, one can also specify the parabolic locus, which we can think of as the toral boundary components and in addition a collection of annuli in the non-toral boundary components which are to be made parabolic. The only condition is that the boundary of any essential annulus in $V$ should not be contained in the parabolic locus. Such a specification is called a pared manifold, see [19] for a precise statement.

Fundamental domains Hyperbolic manifolds can be made by gluing the faces of a polyhedron (or polygon in the surface case) using hyperbolic isometries. The topological condition for the resulting object to be a manifold is that the link of each vertex be a 2 -sphere, see [33]. Thurston's book also gives many examples of interesting gluings. Poincaré's theorem, see [19, 2, 23], tells us that the resulting manifold will be $\mathbb{H}^{3} / G$ for some Kleinian group $G$ iff

- The sum of angles around each edge is $2 \pi$, and

- The resulting manifold with a suitable induced metric is complete.

The construction in $\mathbb{H}^{2}$ is simpler: we glue the sides of a polygon ensuring that the angle sum around each vertex is $2 \pi$. In this case, if the polygon is finite sided there is a simple condition for completeness: Any cycle (that is, sequence of vertices which glue together round the vertex) corresponding to an ideal vertex of the polygon (that is, a vertex where two sides meet on $\partial \mathbb{H}^{2}$ ) is parabolic.

Suppose that conversely we are given a 2- or 3-dimensional hyperbolic manifold. Can we find a corresponding polyhedron? The answer is provided by the Dirichlet domain. The following nice description comes from [23]. Pick a point $a \in M$ and start blowing up a balloon centred at $a$. Eventually one side of the balloon will touch another. If you keep blowing, the parts of the balloon near these touching points will flatten out forming the beginnings of a planar face. As you keep blowing, other points will touch starting further faces. Eventually different faces will meet at vertices. Keep going until you have filled up all of $M$, lift to $\mathbb{H}^{3}$, and you have the 
Dirichlet domain $D_{a}(G)$ centred on $a$. More formally:

$$
D_{a}(G)=\left\{q \in \mathbb{H}^{3}: d(q, a) \leq d(q, g(a)) \text { for all } g \in G \backslash I\right\} .
$$

One can show that $D=D_{a}(G)$ is a convex fundamental polyhedron for the action of $G$ on $\mathbb{H}^{3}$. This means that the images $g(D), g \in G$, tessellate $\mathbb{H}^{3}$, in other words, $g(\operatorname{Int} D) \cap h(\operatorname{Int} D)=\emptyset$ unless $g=h$, and that the images of $D$ cover $\mathbb{H}^{3}$. (Here Int $D$ means the interior of $D$.)

The polyhedron $D$ is locally finite, more precisely only finitely many faces meet any compact set in $\mathbb{H}^{3}$. The faces of $D$ are pieces of the hyperplanes which bisect the distance between $a$ and any of its neighbouring orbit points. They come naturally in pairs: if $D$ meets $g(D)$ along a common face $F$, then $g^{-1}(D)$ meets $D=g^{-1}(g(D))$ along the common face $g^{-1}(F)$. We say that $g^{-1}$ 'pairs' the face $F$ to the face $g^{-1}(F)$, and $g^{-1}$ is called a side pairing of $D$. It is easy to see that if $g^{-1}$ is a side pairing, then so is $g$.

Proposition 2.25. For any $a \in \mathbb{H}^{3}$, a Kleinian group $G$ is generated by the side pairings of $D_{a}(G)$.

Proof. Take $h \in G$ and join $a$ to $h(a)$ by a path $\alpha$ which avoids all vertices of $D=D_{a}(G)$ and its images under $G$. Notice that if $g(D)$ meets $g^{\prime}(D)$ in a common face, then $g^{\prime-1} g(D)$ meets $D$ in a common face so that $g^{\prime-1} g$ is a side pairing. Suppose that the polyhedra crossed by $\alpha$ in order along its path are $D, g_{1}(D), \ldots, g_{k-1}(D), h(D)$. Then $h=\left(I^{-1} g_{1}\right)\left(g_{1}^{-1} g_{2}\right) \ldots\left(g_{k-1}^{-1} h\right)$ expresses $h$ as a product of side pairings.

Corollary 2.26. If for some $a \in \mathbb{H}^{3}$ the Dirichlet domain $D_{a}(G)$ has finitely many faces, then $G$ is finitely generated.

The converse is true in dimension 2 but not in dimension 3 . Groups for which $D_{a}(G)$ has finitely many faces are called geometrically finite and are the subject of the next chapter.

It is high time to have some examples.

Fuchsian groups By definition, a Fuchsian group is a discrete group of isometries of $\mathbb{H}^{2}$. Embedding $\mathbb{H}^{2}$ as a plane in $\mathbb{H}^{3}$, we see 
that any isometry of the plane extends to an isometry of $\mathbb{H}^{3}$. So a Fuchsian group $G$ can be considered as a special case of Kleinian group. The quotient $\mathbb{H}^{2} / G$ is a hyperbolic surface $\Sigma$.

Think of $\mathbb{H}^{2}$ as the equatorial plane in the ball $B^{3}$. Let $G$ be a Fuchsian group and take a fundamental polygon $D_{G}$ for $G$ acting in $\mathbb{H}^{2}$. Now extend each of its sides into a plane in $\mathbb{H}^{3}$. This extends $D_{G}$ to a 'chimney' which forms a fundamental polyhedron for $G$ acting in $\mathbb{H}^{3}$. From this picture, we see that $\mathbb{H}^{3} / G$ is homeomorphic to $S \times(0,1)$, where $S$ is a topological surface homeomorphic to the hyperbolic surface $\Sigma$.

Let us assume that $\Sigma$ is closed or possibly has finitely many punctures, so that $G$ is of the first kind as a Fuchsian group, in other words, its limit set is the whole of $\partial \mathbb{H}^{2}$. Then thinking of $G$ as a Kleinian group acting on $\mathbb{H}^{3} \cup \hat{\mathbb{C}}$, its limit set $\Lambda(G) \subset \hat{\mathbb{C}}$ is the equatorial circle in which $\mathbb{H}^{2}$ meets $\hat{\mathbb{C}}$. The regular set $\Omega$ has two connected components $\Omega^{ \pm}$each of which is simply connected and $G$-invariant. The quotients $\Omega^{ \pm} / G$ each have conformal structures whose corresponding hyperbolic structures are identical with that of $\Sigma$.

Isometries of $\mathbb{H}^{2}$ are exactly the Möbius map which map $\mathbb{H}^{2}$ to itself. An element in $S L(2, \mathbb{C})$ maps $\mathbb{H}^{2}$ to itself iff all its matrix coefficients are real. Thus $I s o m^{+} \mathbb{H}^{2}$ is naturally identified with $S L(2, \mathbb{R}) \subset S L(2, \mathbb{C})$. Conjugating by a Möbius map sends $\mathbb{H}^{2}$ to another disk in $\hat{\mathbb{C}}$. Even though the matrix entries in the conjugated group are no longer real, all the traces remain real. This gives another characterization of a Fuchsian group:

Definition 2.27. A Fuchsian group is a Kleinian group which leaves invariant a disk in $\hat{\mathbb{C}}$. A Kleinian group is Fuchsian iff the traces of all its elements are real.

We can construct lots of examples of Fuchsian groups by starting with a finite sided hyperbolic polygon, pairing sides in some specified way, and checking the conditions of the Poincaré theorem. For example, identifying opposite sides of a regular $4 g$-gon with interior angle $\pi / 2 g$, we obtain a hyperbolic structure on a closed surface of genus $g$ whose associated Fuchsian group is generated by the isometries which pair the opposite sides. 
Quasifuchsian groups By definition, a quasifuchsian group is a quasi-isometric or quasiconformal deformation of a Fuchsian group. We shall see in more detail what this means in the next chapter. For the moment, let's just assume we have a Fuchsian group $\Gamma$, a Kleinian group $G$, a group isomorphism $\chi: \Gamma \rightarrow G$ and a homeomorphism $\psi: \hat{\mathbb{C}} \rightarrow \hat{\mathbb{C}}$ such that $\psi(g z)=\chi(g) \psi(z)$ for all $z \in \hat{\mathbb{C}}$ and $g \in \Gamma$. It is a deep result of Marden [22] that $\psi$ can always be taken to be quasiconformal, see Definition 3.39.

From the construction, the limit set of a quasifuchsian group is always a topological circle and the regular set has two simply connected $G$-invariant components $\Omega^{ \pm}$. The 3 -manifold $\mathbb{H}^{3} / G$, being homeomorphic to $\mathbb{H}^{3} / \Gamma$, is homeomorphic to $S \times(0,1)$. However the hyperbolic or conformal structures on the two components $\Omega^{ \pm} / G$ are now different. The following is a famous result of Bers [3]:

Theorem 2.28 (Simultaneous Uniformisation). Given any two conformal structures $\omega^{ \pm}$on $\Sigma$, there exists a unique quasifuchsian group for which $\Omega^{ \pm} / G$ have the structures $\omega^{ \pm}$. (Here 'unique' means 'unique up to conjugation in $S L(2, \mathbb{C})$ '.)

The limit set of a quasifuchsian group is a fractal:

Theorem 2.29. (Bowen) Suppose that $G$ is quasifuchsian but not Fuchsian. Then the Hausdorff dimension of $\Lambda(G)$ is strictly greater than 1.

You can find many pictures of limit sets of quasifuchsian groups in [31].

Schottky groups Take $2 k$ pairwise disjoint round disks $E_{i}, E_{i}^{\prime}, i=$ $1, \ldots, k$ in $\hat{\mathbb{C}}$ and choose $e_{i} \in S L(2, \mathbb{C})$ such that $e_{i}$ maps the inside of $E_{i}$ to the outside of $E_{i}^{\prime}$. The group $G$ generated by $e_{1}, \ldots, e_{k}$ is called a (classical) Schottky group. If we place a hemisphere over each disk, then we can view the region $D$ in $\mathbb{H}^{3}$ outside all the hemispheres as a polyhedron in $\mathbb{H}^{3}$. The $e_{i}$ match the sides of $D$ in pairs and $D$ is a fundamental domain for $G$ acting in $\mathbb{H}^{3}$. It follows from Poincaré's theorem that $G$ is free and discrete. Klein gave a nice direct proof of this fact, the simplest case of Klein's combination theorem, often called the ping-pong theorem, see [17] or [6]. It is good exercise to 
convince oneself that the 3-manifold obtained by gluing the faces of $D$ using the side pairings is a genus $k$ handlebody.

As explained in great detail in [31], each 'infinite reduced word' in the generators in $G$ corresponds to a nested sequence of images of the disks $E_{i}, E_{i}^{\prime}$. Each limit point is the infinite intersection of such a nested sequence. It follows that the limit set $\Lambda(G)$ is a Cantor set and the ordinary set $\Omega$ has a single $G$-invariant component which is not simply connected. It is another good exercise to find which curves in $\Omega$ correspond to compressing disks in $\mathbb{H}^{3} / G$.

Any Kleinian group has lots of Schottky subgroups. This is based on the fact that sufficiently high powers of loxodromics have disjoint isometric circles which serve as the disks from which to construct the group. Based on this we find:

Proposition 2.30. (See [26] Theorem 2.9) $G$ is non-elementary iff it contains a free subgroup on 2 generators.

Just like Fuchsian groups, Schottky groups can be deformed using homeomorphisms of $\hat{\mathbb{C}}$. This does not change the topology of the 3manifold. Such groups are also known as (non-classical) Schottky groups. In fact we have:

Theorem 2.31. (Maskit, see [26] Theorem 4.23) The following are equivalent:

1. $M_{G}$ is a handlebody.

2. $G$ is Schottky.

3. $G$ is free and purely loxodromic.

Combination theorems These are important theorems which enable one to build up complicated groups from simple ones by gluing along surfaces in the boundary. The fundamental principal is the same as that in the ping-pong theorem. We don't have time to go into this here; good accounts can be found in $[23,26,19]$ and for full details see [25]. 


\section{Chapter 3: Geometrically finite groups}

In two dimensions, there are many possible hyperbolic structures on a closed surface of genus $g \geq 2$. The space of possible such structures on a given topological surface is known as Teichmüller space. By contrast, in dimension 3 or higher the Mostow rigidity theorem says that a closed hyperbolic 3-manifold is rigid, more precisely that for $n \geq 3$, any two finite volume hyperbolic $n$-manifolds with isomorphic fundamental groups are isometric. In dimension 3 this means that any isomorphism between holonomy groups is actually a conjugacy in $\operatorname{PSL}(2, \mathbb{C})$.

In these notes we are mainly interested in hyperbolic 3-manifolds with infinite volume. In general such manifolds are not rigid. In this chapter we study an important class of such hyperbolic manifolds, the geometrically finite manifolds, whose deformation theory is central to the general case. As a result of the work of Ahlfors, Bers and Marden in the 1960's and 70's, we understand that their deformations can be completly described in terms of the deformations of the conformal boundary at infinity.

\section{Geometrically finite groups}

Definition 3.32. A Kleinian group is geometrically finite if it has a finite sided fundamental domain.

As we saw in Corollary 2.26, the side pairings generate the group, so a geometrically finite group is always finitely generated.

TheOREM 3.33. A Fuchsian group is finitely generated iff it is geometrically finite.

For the proof, see [2]. As was pointed out by Greenberg, Theorem 3.33 fails in dimension 3, see Theorem 4.56. Marden systematically investigated geometrically finite groups in [22]. The first point is to understand the cusps, for which we need the idea of pairing tubes as discussed following Theorem 2.23.

Proposition 3.34. (Marden) If $G$ is geometrically finite then there are only finitely many cusps. The rank 1 cusps come in pairs; each pair corresponds to a pairing tube which matches two punctures on $\Omega / G$. 
This leads easily to

Theorem 3.35. (Marden) $G$ is geometrically finite iff $M_{G}^{0}$ is compact.

Here $M_{G}^{0}$ is the Kleinian manifold $M_{G}=\left(\mathbb{H}^{3} \cup \Omega\right) / G$ with cusp neighbourhoods removed, as explained in Chapter 2. Proofs can also be found in [26].

Definition 3.36. $G$ is (topologically) tame iff $\mathbb{H}^{3} / G$ is homeomorphic to the interior of a compact 3-manifold.

TheOREM 3.37. (Marden) A geometrically finite group $G$ is tame.

Marden conjectured that if $G$ is finitely generated, then $G$ is tame, see Conjecture 4.53.

Ahlfors-Bers deformation theory Let us examine in more detail the assertion that the deformations of geometrically finite groups are completely described in terms of the deformations of the conformal boundary $\Omega / G$. General references for this discussion are as usual $[26,19,23]$.

Definition 3.38. Let $\left(X_{1}, d_{1}\right)$ and $\left(X_{2}, d_{2}\right)$ be metric spaces. $A$ homeomorphism $f: X_{1} \rightarrow X_{2}$ is a quasi-isometry if there exists $k>0$ such that

$$
d_{1}(p, q) / k \leq d_{2}(f(p), f(q)) \leq k d_{1}(p, q)
$$

for all $p, q \in X_{1}$.

Definition 3.39. Let $U \subset \hat{\mathbb{C}}$ be an open set. A homeomorphism $f: U \rightarrow f(U) \subset \hat{\mathbb{C}}$ is quasiconformal if

$$
K(z)=\lim _{r \rightarrow 0} \frac{\max _{\theta}\left|f\left(z+r e^{i \theta}\right)-f(z)\right|}{\min _{\theta}\left|f\left(z+r e^{i \theta}\right)-f(z)\right|}
$$

is bounded on $U$. It is called $K$-quasiconformal if $K(z) \leq K$ for almost all $z \in U$. The dilatation $K_{f}$ of $f$ is the infimum of $K$ for which $f$ is $K$-quasiconformal. 
Proposition 3.40. Every quasi-isometry $\mathbb{H}^{3} \rightarrow \mathbb{H}^{3}$ extends to a quasiconformal homeomorphism of $\hat{\mathbb{C}}$.

Suppose that $G_{1}, G_{2}$ are Kleinian groups and that $\chi: G_{1} \rightarrow G_{2}$ is an isomorphism. A quasiconformal map $\psi: \Omega\left(G_{1}\right) \rightarrow \Omega\left(G_{2}\right)$ is said to induce $\chi$ if $\psi(g z)=\chi(g) \psi(z)$ for all $g \in G_{1}$ and $z \in \hat{\mathbb{C}}$. The isomorphism $\chi$ is called type preserving if $\chi(g)$ is parabolic in $G_{2}$ if and only if $g$ is parabolic in $G_{1}$. It is possible to have a quasiconformal map $\psi: \Omega\left(G_{1}\right) \rightarrow \Omega\left(G_{2}\right)$ which induces an isomorphism $\chi: G_{1} \rightarrow G_{2}$ but for which there is no corresponding quasi-isometry $\mathbb{H}^{3} / G_{1} \rightarrow \mathbb{H}^{3} / G_{2}$. (Examples are provided by groups on the boundary of Schottky space, see [26] page 120.) However we have:

Theorem 3.41 (MARden's Isomorphism theorem [22]). Let $G_{1}$ be a geometrically finite group with $\partial M_{G}^{0} \neq \emptyset$. Suppose that $\chi$ : $G_{1} \rightarrow G_{2}$ is a type preserving isomorphism induced by an orientation preserving quasiconformal map $\psi: \Omega\left(G_{1}\right) \rightarrow \Omega\left(G_{2}\right)$. Then:

1. $G_{2}$ is geometrically finite.

2. $\psi$ extends to a quasi-isometry $\mathbb{H}^{3} / G_{1} \rightarrow \mathbb{H}^{3} / G_{2}$.

3. $\psi$ extends to a quasiconformal homeomorphism of $\hat{\mathbb{C}}$.

4. If $\psi$ is conformal on $\Omega\left(G_{1}\right)$ then it is Möbius, so that $\mathbb{H}^{3} / G_{1}$ is isometric to $\mathbb{H}^{3} / G_{2}$.

Notice that (4) is closely related to the Mostow rigidity theorem, in the proof of which quasi-isometries play a central role.

This result reduces the problem of understanding deformations of hyperbolic structures on a geometrically finite manifolds to that of understanding the structures on $\Omega / G$. The following extension of Bers' simultaneous uniformisation theorem 2.28 describes the deformation theory of geometrically finite groups.

Theorem 3.42. (See $[26,19]$ ) Let $M=\mathbb{H}^{3} / G$ be a geometrically finite hyperbolic 3-manifold with incompressible boundary. Then the quasi-isometric deformation space $\mathcal{Q I}(M)$ of $M$ is $\Pi_{i} \operatorname{Teich}\left(S_{i}\right)$, where the product runs over the surfaces $S_{i}$ making up $\Omega / G$. 
If $S_{0}$ is a topological surface, the Teichmüller space Teich $\left(S_{0}\right)$ describes the possible conformal structures on the marked surface $S_{0}$. More precisely, a point in $\operatorname{Teich}\left(S_{0}\right)$ is a pair $(S, \phi)$ such that $S$ is Riemann surface and $\phi: S_{0} \rightarrow S$ is a homeomorphism. Pairs $(S, \phi),\left(S^{\prime}, \phi^{\prime}\right)$ are equivalent if there is a conformal map $f: S \rightarrow S^{\prime}$ homotopic to $\phi^{\prime} \phi^{-1}$. One of Teichmüller's main theorems states that for any two points $\left(S_{1}, \phi_{1}\right),\left(S_{2}, \phi_{2}\right) \in \operatorname{Teich}\left(S_{0}\right)$, there is a unique quasiconformal map $S_{1} \rightarrow S_{2}$ homotopic to $\phi_{1} \phi_{2}^{-1}$ which minimises the dilatation, called the extremal map. The log of the dilatation provides a metric on Teich $\left(S_{0}\right)$. Teichmüller also showed that if $S_{0}$ has genus $g$ with $b$ punctures, then Teich $\left(S_{0}\right)$ is homeomorphic to $\mathbb{R}^{6 g-6+2 b}$. There are many books on Teichmüller theory. Brief summaries suitable to our viewpoint can be found in [30, 19].

The quasi-isometric deformation space $\mathcal{Q I}(M)$ of a 3-manifold $M$ can be defined in a similar way, see [26]. We always need the base manifold $M$ (or base surface $S_{0}$ ) to keep track of the marking on $M$; otherwise, we might be seeing the same structure on a surface which differed from the original one by a diffeomorphism which was not homotopic to the identity. (This is why we restricted to incompressible boundary in the statement of Theorem 3.42.)

Triply punctured spheres Note that if $S_{0}$ is a sphere with 3 punctures (so $g=0$ and $b=3$ ) then $6 g-6+2 b=0$. This means that $S_{0}$ is rigid. In other words, it carries a unique hyperbolic structure which cannot be deformed. In fact it is not hard to prove the following lemma, see [31] Note 7.1:

Lemma 3.43. Suppose that $A, B \in S L(2, \mathbb{C})$ are parabolic and that $A B$ is also parabolic. Suppose their fixed points are all distinct. Then there exists $C \in S L(2, \mathbb{C})$ such that $C A C^{-1}=\left(\begin{array}{ll}1 & 2 \\ 0 & 1\end{array}\right)$ and $C B C^{-1}=\left(\begin{array}{cc}1 & 0 \\ -2 & 1\end{array}\right)$.

Convince yourself that this implies that $S_{0}$ is rigid!

Convergence of Kleinian groups There are several different ways in which one might say that Kleinian groups are close. The 
topology implied in Theorem 3.42 is essentially that of GromovHausdorff convergence. It is important to specify basepoints.

Roughly, hyperbolic manifolds $M, M^{\prime}$ with basepoints $x \in M, x^{\prime} \in$ $M^{\prime}$ are close if for large $r$, the $r$-balls in $M, M^{\prime}$ centred on $x, x^{\prime}$ look 'almost' the same. A more formal definition is that of polyhedral convergence ${ }^{3}$ :

Definition 3.44. Let $G_{n}$ be a sequence of Kleinian groups. Then $G_{n}$ converges polyhedrally to the Kleinian group $H$ if the Dirichlet domains $D_{a}\left(G_{n}\right)$ with base point a $\in \mathbb{H}^{3}$ converge to the Dirichlet domain $D_{a}(H)$, uniformly on compact sets in $\mathbb{H}^{3}$, and if in addition the side pairing transformations of $D_{a}\left(G_{n}\right)$ converge to those of $D_{a}(H)$ in the following sense:

(i) Each face pairing of $D_{a}(H)$ is the limit of face pairings of $D_{a}\left(G_{n}\right)$ and

(ii) The limit of any convergent subsequence of face pairings of $D_{a}\left(G_{n}\right)$ is a face pairing of $D_{a}(H)$.

This leads to:

Definition 3.45. Let $G_{n}$ be a sequence of Kleinian groups. Then $G_{n}$ converges geometrically to the Kleinian group $H$ if:

(i) For each $h \in H$, there is a sequence $g_{n} \in G_{n}$ which converges to $h$ and

(ii) If a subsequence $g_{n_{r}} \in G_{n_{r}}$ converges to $\gamma \in S L(2, \mathbb{C})$, then $\gamma \in H$.

Polyhedral and geometric convergence turn out to be the same and, modulo a discussion about base points, and conjugation in $S L(2, \mathbb{C})$, are the same as Gromov-Hausdorff convergence of the corresponding manifolds. It is also the same as convergence of the limit sets:

TheOREm 3.46. (Kerckhoff-Thurston, see [26, 23]) Suppose that the groups $G_{n}$ are geometrically finite and boundary incompressible, and suppose that $G_{n}$ converge geometrically to $H$. Then $\Lambda\left(G_{n}\right)$ converges to $\Lambda(H)$ in the sense of Hausdorff convergence of closed subsets of $\hat{\mathbb{C}}$.

\footnotetext{
${ }^{3}$ General references for this section are [26, 19, 23, 32].
} 
There is also a formulation in terms of convergence of regular sets, see for example [26, 23].

There is another definition of convergence which is more natural if we are thinking in terms of groups of matrices:

DeFinition 3.47. Let $\Gamma$ be a fixed abstract group (think $\Gamma=\pi_{1}(M)$ ). Suppose we have a sequence of homomorphisms $\rho_{n}: \Gamma \rightarrow G_{n}$ where $G_{n}$ is Kleinian. Suppose that for each $\gamma \in \Gamma$, the sequence $\rho_{n}(\gamma)$ converges to a Möbius map $\rho_{\infty}(\gamma)$. Then $G_{n}$ converges algebraically to the group $G_{\infty}=\left\{\rho_{\infty}(\gamma): \gamma \in \Gamma\right\}$. The group $G_{\infty}$ is called the algebraic limit of the $G_{n}$.

It is an important and non-obvious fact that the algebraic limit of discrete groups is discrete. More precisely:

Theorem 3.48. (Chuckrow) Suppose that $\Gamma$ is a non-elementary Kleinian group and that each $\rho_{n}$ is an isomorphism to a Kleinian group $G_{n}$. Suppose that $G_{n}$ converges algebraically to $G_{\infty}$. Then $G_{\infty}$ is Kleinian and non-elementary and $\rho_{\infty}$ is an isomorphism.

Proof. This follows from several applications of Jørgensen's inequality Theorem 1.8. For example, if $\rho_{\infty}$ is not injective, there exists $g \in \Gamma$ with $\rho_{\infty}(g)=I$. Pick a loxodromic $h \in \Gamma$ such that $g, h$ have distinct fixed points. Then $\left\langle\rho_{n}(g), \rho_{n}(h)\right\rangle$ is non-elementary and $\rho_{n}(g) \rightarrow I$. So

$$
\left|\operatorname{Tr}^{2} \rho_{n}(g)-4\right|+\left|\operatorname{Tr} \rho_{n}(g) \rho_{n}(h) \rho_{n}(g)^{-1} \rho_{n}(h)^{-1}-2\right| \rightarrow 0
$$

which is impossible.

The relationship between algebraic and geometric convergence is rather subtle.

Theorem 3.49. (Jørgensen-Marden) Suppose that $G_{n}$ converges to $G_{\infty}$ algebraically. Then there exists a subsequence $G_{n_{r}}$ which converges geometrically to a Kleinian group $H$. Moreover any geometric limit of any subsequence of the groups $G_{n}$ contains $G_{\infty}$.

If $G_{\infty}$ and $H$ are finitely generated, then there is a sequence of surjective homomorphisms $\psi_{n}: H \rightarrow G_{n}$ such that $\lim \psi_{n}(h)=h$ for all $h \in H$. 
The best situation is that in which $G_{n}$ converges both algebraically and geometrically to the same limit. In this case the convergence is called strong. In general the algebraic and geometric limits may not agree. What happens is that 'extra' parabolics appear in the limit. Roughly, one can have a sequence of loxodromics $h_{n}$ converging to a parabolic $h$ such that certain powers $h_{n}^{m_{n}}$ also converge to a parabolic $h^{\prime}$ with the same fixed point but with a different translation direction. This mechanism is described in various places, for example [26]. There is an explicit example, with pictures, in [31] P. $340 \mathrm{ff}$. Kerckhoff and Thurston produced a famous example in which the algebraic and geometric limits differ, on which much subsequent work is based.

It seems likely that the apperance of extra parabolics is the only reason the limit may not be strong:

Theorem 3.50. Suppose that $\rho_{n}(\Gamma)$ converges to $G_{\infty}=\rho_{\infty}(\Gamma)$ algebraically. The convergence is strong if either:

(i) $\rho_{n}(\Gamma)$ is geometrically finite for each $n$ and there are no new parabolics (ie $g \in G_{\infty}$ is parabolic iff $\rho_{n}(g) \rho_{\infty}^{-1}(g)$ is parabolic for each $n$ ) or

(ii) There are no new parabolics and $\Omega\left(G_{\infty}\right) \neq \emptyset$.

A lot of effort has gone into proving that various properties persist in the algebraic limit. The following result is the outcome of many years' work by Thurston, Brock, Bromberg, Canary, Evans, Ohshika, Souto and others:

THEOREM 3.51. ([9]) The algebraic limit of geometrically finite groups is tame.

Cusp groups Here is a much easier result which we shall need in the next chapter. It is a simple consequence of Theorem 3.41.

Theorem 3.52. Suppose that $G_{n}=\rho_{n}\left(G_{0}\right)$ is a sequence in $\mathcal{Q I}(M)$ for some geometrically finite manifold $M=\mathbb{H}^{3} / G_{0}$. Suppose also that the algebraic limit $\rho_{\infty}\left(G_{0}\right)$ is geometrically finite but not in $\mathcal{Q I}(M)$. Then there is an element $g \in G_{0}$ such that $\rho_{n}(g)$ is loxodromic but $\rho_{\infty}(g)$ is parabolic. 
Such groups are called cusp groups, because a geodesic loop which had some definite length in $\mathbb{H}^{3} / G_{0}$ has been 'pinched' to become parabolic. These new parabolics all correspond to rank 1 cusps. The existence of cusp groups was first proved by Bers and Maskit using sequences of quasiconformal deformations. Any 'reasonable' collection of curves on the boundary of a hyperbolic 3-manifold can be pinched in this way. If there are no more loops which can be pinched, the group is called maximally parabolic. Each component of the boundary of such a group is either a cusp torus or a triply punctured sphere. The convex core of such a group (see Chapter 4 for a definition) has totally geodesic boundary. Such groups are rigid: to see this, double across the triply punctured spheres in the boundary and apply Mostow rigidity. The limit set of each triply punctured sphere group is a circle and every component of $\Omega$ is a round disk.

If $G$ is quasifuchsian, then $\mathbb{H}^{3} / G$ is homeomorphic to $S \times(0,1)$ for some topological surface $S$. Choose a maximal set of pairwise disjoint non-homotopic curves on $S$ (a pants decomposition). All these curves can be made parabolic by pinching the corresponding loops on $\Omega^{-} / G$. The other boundary surface $\Omega^{+} / G$ remains unpinched and homeomorphic to $S$. By Theorem 3.42, the deformation space of this family of groups is exactly Teich $S$. One can parameterise it as a $(3 g-3+2 b)$ complex dimensional subspace of the space of all representations $\pi_{1}(S) \rightarrow S L(2, \mathbb{C})$. This is called the Maskit embedding of Teichmüller space. If $S$ is a torus with one puncture, the Maskit embedding has complex dimension one. This is examined in great detail in [31].

\section{Chapter 4: Geometrically infinite groups}

What can one say about groups which are not geometrically finite? For many years their full classification was a mystery, but as a result of remarkable work over the last few years, we now have an essentially complete picture. At the time of writing, many of the results are very new and not all are fully published. Notwithstanding, [23] is an excellent source.

Recall from the last chapter that a group is called tame if $\mathbb{H}^{3} / G$ is homeomorphic to the interior of a compact 3-manifold. As we saw, 
any geometrically finite group is tame.

CONJECTURE 4.53 (MARDEN'S TAMENESS CONJECTURE/THEOREM). Every finitely generated group is tame.

Conjecture 4.54 (Bers' Density COnjecture/theorem). Every Kleinian group is an algebraic limit of geometrically finite groups.

CONJECTURE 4.55 (ENDING LAMINATION CONJECTURE/THEOREM). Every tame group is determined up to isometry by its 'end invariants'.

The meaning of 'end invariants' will be explained below.

These conjectures are now all essentially proved. The tameness conjecture was done independently by Agol [1] and Calegari and Gabai [10]. The ending lamination conjecture was proved in many special cases by Minsky and finally completed by Brock, Canary and Minsky [12]. More or less simultaneously, Bromberg introduced some beautiful ideas to prove Conjecture 4.54 in special cases and under the hypothesis of tameness. This was extended to the general incompressible boundary case in [7]. A completely different proof follows by combining Conjectures 4.53 and 4.55, see [9].

Our starting point is a geometrically finite group $G$ with corresponding manifold $M=\mathbb{H}^{3} / G$. What are all the groups which can be obtained as algebraic limits of groups in the quasi-isometric deformation space $\mathcal{Q I}(M)$ ? We think of $\mathcal{Q I}(M)$ as embedded in the larger space of representations $\pi_{1}(M) \rightarrow S L(2, \mathbb{C}) .{ }^{4}$ The space of representations, taken up to conjugacy in $S L(2, \mathbb{C})$, turns out to be a smooth complex variety $\mathcal{R}(M)$ of the 'expected' dimension, namely the sum of the dimensions of the Teichmüller spaces associated to the components of $\partial M_{G}$. It follows from the simultaneous uniformisation theorem 3.42 that $\mathcal{Q I}(M)$ is an open subset of $\mathcal{R}(M)$.

Theorem 3.52 asserts that the geometrically finite groups on $\partial \mathcal{Q I}(M)$ are the cusp groups formed by pinching one or more loxodromic elements until they become parabolic. This leads to:

THEOREM 4.56. (Greenberg) There exist Kleinian groups which are not geometrically finite.

\footnotetext{
${ }^{4}$ We have to be careful to specify whether or not the representations should be type preserving, see for example [19] for details.
} 
Proof. A cusp group in which the element $g$ is parabolic lies in the subvariety of $\mathcal{R}(M)$ on which $\operatorname{Tr}^{2} g=4$. Since there are only countably many possible loops which can be pinched to make cusps, and since each subvariety has complex codimension 1 , the union of these sets cannot be all of $\partial \mathcal{R}(M)$.

Here is a famous recent result, conjectured by Bers and originally proved in a special case by McMullen.

Theorem 4.57. ([18, 13]) Cusp groups are dense on $\partial \mathcal{Q I}(M)$.

The boundary $\partial \mathcal{Q I}(M)$ is a fascinating object which sadly we do not have time for here. It appears to have complicated fractal structure. For pictures and an account of the boundary of the Maskit embedding of Teichmüller space for the once punctured torus, see [31].

Given that the geometrically finite groups on $\partial \mathcal{Q I}(M)$ are produced by pinching simple closed curves, what about the rest? Thurston had the wonderful idea that the remaining groups on $\partial \mathcal{Q I}(M)$ could be produced by 'pinching' some other objects which in a certain sense 'complete' the space of simple closed curves on $\partial M_{G}$. The objects in question are projective measured laminations, which Thurston used to compactify Teichmüller space. We digress to explain very briefly what these are.

Geodesic laminations A standard reference for this section is [20], see also $[15,32]$. There is a good brief summary in the appendix of $[30]$.

Let $S$ be a hyperbolic surface. A geodesic (not necessarily closed) on $S$ is simple if it does not intersect itself. A geodesic lamination on $S$ is a closed set which is the disjoint union of simple geodesics called its leaves. Such a lamination forms a partial foliation of the surface. For example, it might consist of finitely many pairwise disjoint simple closed geodesics. More typically, however, a transversal to a lamination intersects the lamination in a Cantor set and the components of the complement are ideal polygons. A lamination is arational if all complementary components are ideal triangles or punctured bigons. A result of Birman and Series says that any lamination has Hausdorff dimension 1. 
A measured geodesic lamination is a geodesic lamination together with a tranverse invariant measure. That means, an assignment of a (finite Borel) measure to each transversal, which is invariant under the 'push forward' map along leaves. For example, a closed simple geodesic $\gamma$ has an associated transverse measure $\delta_{\gamma}$ which assigns to a transversal $T$ the measure $i(T, \gamma)$, ie the number of times $T$ intersects $\gamma$. More generally, we shall call a lamination rational ${ }^{5}$ if it is a sum $\sum_{i} a_{i} \delta_{\gamma_{i}}$ where $\gamma_{i}$ are pairwise disjoint closed geodesics and $a_{i}>0$. Any transverse measure can be scaled by multiplying by positive scalar. This defines an equivalence relation on measured laminations. The equivalence classes are called projective measured laminations.

Let $\mathcal{M L}$ denote the space of measured laminations on $S$ with the weak topology. That is, a sequence $\nu_{n} \in \mathcal{M L}$ converges to $\nu \in \mathcal{M L}$ iff $\nu_{n}(T)$ converges to $\nu(T)$ for every transversal $T$. The space $\mathcal{M L}$ turns out to be the completion of the rational laminations in this topology. Thurston showed that $\mathcal{M L}(S)$ is a ball of (real) dimension $6 g-6+2 b$. This dimension is no coincidence! The following remarkable result of Thurston shows that Teichmüller space can be compactified by adjoining the space of projective laminations $\mathcal{P} \mathcal{M L}(S)$, see $[20,30]$.

Theorem 4.58. Suppose that $\omega_{n} \in \operatorname{Teich}(S)$. Then either:

(i) A subsequence $\omega_{n_{r}}$ converges to a point $\omega_{\infty} \in \operatorname{Teich}(S)$ or

(ii) There exist $c_{n}>0$ with $c_{n} \rightarrow 0$ and $\xi \in \mathcal{M L}$ such that

$$
c_{n} l_{\omega_{n}}(\gamma) \rightarrow i(\gamma, \xi)
$$

for every simple curve $\gamma$ on $S$.

Here $l_{\omega}(\gamma)$ is the hyperbolic length of $\gamma$ in the hyperbolic surface whose associated conformal structure is $\omega$, and $i(\gamma, \xi)$ denotes intersection number; it is the continuous linear extension of the geometric intersection number between geodesics to $\mathcal{M L}$. Since the second condition is unchanged if we scale everything by $a>0$, the limit $\xi$ really only depends on its projective class $[\xi]$. We say that $\omega_{n}$ converges to $[\xi]$.

\footnotetext{
${ }^{5}$ This is not universally accepted terminology. It is not the opposite of arational.
} 
As a special case, suppose that $\omega_{n} \rightarrow[\xi]$ in such a way that along the sequence $\omega_{n}$, the length $l_{\omega_{n}}(\gamma)$ of a curve $\gamma$ stays bounded. Then, since $c_{n} \rightarrow 0$, we have $c_{n} l_{\omega_{n}}(\gamma) \rightarrow 0$. This means that $i(\gamma, \xi)=0$, which means that either $\gamma$ is disjoint from $\xi$ or that $\xi$ contains $\gamma$ as a closed leaf. This suggests that if we degenerated $S$ by pinching $\gamma$, the structures on $S$ would converge to $\left[\delta_{\gamma}\right] \in \mathrm{P} \mathcal{M L}$. (To make a correct formal statement we also have to control the degeneration of $S \backslash \gamma \cdot)$

Recall that a quasifuchsian group is uniquely specified by the structures $\omega^{ \pm}$on the two components $\Omega^{ \pm} / G$. Denote this group $G\left(\omega^{+}, \omega^{-}\right)$.

Theorem 4.59 (Thurston's Double limit theorem). Suppose that $\rho_{n}: \pi_{1}(S) \rightarrow S L(2, \mathbb{C})$ is a sequence of representations so that $\rho_{n}\left(\pi_{1}(S)\right)$ is the quasifuchsian group $G\left(\omega_{n}^{+}, \omega_{n}^{-}\right)$, and suppose that $\omega_{n}^{ \pm}$converge to points $[\xi]^{ \pm} \in \mathcal{P} \mathcal{M L}$. Suppose also that $\xi^{ \pm}$are arational and that $i\left(\xi^{+}, \xi^{-}\right) \neq 0$. Then $\rho_{n}$ has a subsequence which converges algebraically to a geometrically infinite group. The limit group is doubly degenerate and has ending laminations $\xi^{+}, \xi^{-}$.

We will explain the meaning of the last statement below.

The convex core Before getting to geometrically infinite groups, it is also useful to look at the convex core of $\mathbb{H}^{3} / G$. By definition, this is the smallest closed convex subset of $\mathbb{H}^{3} / G$ containing all closed geodesics. Alternatively, let $\mathcal{C}(G)$ be the convex hull in $\mathbb{H}^{3}$ of the limit set $\Lambda(G)$, sometimes called the Nielsen region of $G$. The convex core of $\mathbb{H}^{3} / G$ is just $\mathcal{C}(G) / G$. The Nielsen region is $\mathbb{H}^{3}$ iff $\Lambda(G)=\hat{\mathbb{C}}$. Various people have made nice pictures of convex cores; one such picture, made by Minsky, is reproduced as Figure 12.6 in [31].

Geometrically finite groups can be neatly characterised in terms of their convex core, see for example [26]:

Proposition 4.60. A non-Fuchsian group Kleinian group $G$ is geometrically finite iff its convex core has finite volume.

The restriction to non-Fuchsian groups is because the convex core of a Fuchsian group is contained in a single hyperbolic plane, so always has zero volume even if the group is infinitely generated. 
A Fuchsian group is geometrically finite iff its Nielsen region has finite 2-dimensional area. The Nielsen region equals $\mathbb{H}^{2}$ unless the group is of the second kind, that is, $\Lambda(G)$ is a proper subset of the circle $\partial \mathbb{H}^{2}$. In this case, and assuming that $G$ is finitely generated, the Nielsen region is bounded by axes of hyperbolic elements which project to closed geodesics which cut off the infinite ends or funnels on the quotient surface $\mathbb{H}^{2} / G$.

In 3-dimensions, the boundary of the convex core is a union of surfaces. There is a nice retraction map $r: \mathbb{H}^{3} \cup \Omega \rightarrow \mathcal{C}$. If $x \in \mathcal{C}$, then $r(x)=x$. To define $r(x)$ for a point $x \in \mathbb{H}^{3} \cup \Omega$ outside $\mathcal{C}$, blow out expanding balloons (spheres if $x \in \mathbb{H}^{3}$ and horoballs if $x \in \Omega)$. Then $r(x)$ is the point at which you first hit $\mathcal{C}$. Since $\mathcal{C}$ is convex, $r(x)$ is well defined. It is pretty obviously continuous and $G$ invariant. In fact, $r$ can be modified to a homeomorphism between each component of $\Omega / G$ and the component of $\partial \mathcal{C} / G$ which it 'faces'.

Thurston showed that $\partial \mathcal{C} / G$ has much more structure. It is an example of a pleated surface:

Definition 4.61. A pleated surface in a hyperbolic 3 manifold $M$ consists of a hyperbolic surface $\Sigma$, a geodesic lamination $\lambda$ on $\Sigma$, and a map $f: \Sigma \rightarrow M$ such that:

(i) $f$ is an isometry between the given metric on $\Sigma$ and the induced metric on $f(M)$.

(ii) The restriction of $f$ to each leaf of $\lambda$ is an isometry to a geodesic in $\mathbb{H}^{3}$.

(iii) Each component of $\Sigma \backslash \lambda$ maps isometrically to a piece of totally geodesic plane in $M$.

The images of the leaves of $\lambda$ are called the bending lines of the pleated surface. Roughly speaking, you can think of a pleated surface as a bent surface in $\mathbb{H}^{3} / G$ whose lift to $\mathbb{H}^{3}$ rolls out onto $\mathbb{H}^{2}$ to give the hyperbolic metric on $\Sigma$. Pleated surfaces are explained in detail in [15]. They play a key role in the proof of the ending lamination conjecture.

TheOREM 4.62. (Sullivan, see [15]) If $\partial M$ is incompressible, then there is a universal bound on the Teichmüller distance between the hyperbolic metrics on $\partial \mathcal{C} / G$ and on the corresponding components of $\Omega / G$. 
Geometrically infinite ends The formal definition of an end of a general open 3-manifold $M$ is a bit messy. It is simplified when $M$ has a compact core: this is a compact submanifold $M_{c}$ such that the inclusion $M_{c} \rightarrow M$ is a homotopy equivalence. It is a deep result of Scott, that any 3-manifold with finitely generated fundamental group has a compact core. This was refined by McCullough to show that if $M$ is hyperbolic, one can choose the core to have a 'standard' shaped boundary in the neighbourhood of cusps. This is called a relative compact core, see for example [19] for details.

We can now define an end of $M$ to be a component of $M \backslash M_{c}$. The ends are in bijective correspondence with the components of $\partial M_{c}$. An end is topologically tame if it has a neighbourhood $U$ which is homeomorphic to $S \times[0,1)$ for some surface $S$; it is incompressible if $S$ is incompressible. The manifold $M$ is called boundary incompressible if every component of $\partial M_{c}$ is incompressible.

Recall that $M$ is tame if it is homeomorphic to the interior of a compact 3-manifold. We have:

Lemma 4.63. $M$ is tame iff each of its ends are tame.

If $M$ is geometrically finite without cusps, then the retraction $r$ provides a homotopy equivalence between $\mathbb{H}^{3} / G$ and the compact manifold $\mathcal{C} / G$. This can easily be modified in case of cusps to a retraction to the compact manifold $\left(\mathcal{C} \cap M_{G}^{0}\right) / G$. Thus we can take $\left(\mathcal{C} \cap M_{G}^{0}\right) / G$ to be the core, so the ends correspond bijectively to components of $\partial \mathcal{C} / G$. In general, an end is called geometrically finite if it contains a neighbourhood which is disjoint from $\mathcal{C} / G$ and geometrically infinite otherwise. Thurston and Bonahon described the structure of geometrically infinite incompressible ends.

Definition 4.64. A sequence of closed geodesics $\gamma_{n}$ exits the end $E$ if $\gamma_{n} \subset E$ and if only finitely many $\gamma_{n}$ intersect any compact set $K \subset M$.

Here is the key theorem:

Theorem 4.65. (Bonahon [5]) Let $M$ be a boundary incompressible hyperbolic 3-manifold. Let $E$ be a geometrically infinite end corresponding to a component of $\partial M_{c}$ homeomorphic to a surface $S$. Then there exists a sequence of closed geodesics $\gamma_{n}$ which exit E. The $\gamma_{n}$ 
can be taken to be homotopic to simple closed curves on $S$. Moreover the projective measured laminations $\left[\delta_{\gamma_{n}}\right]$ converge to a measured lamination $[\lambda] \in \mathcal{P} \mathcal{M L}(\mathcal{S})$. The underlying support of $\lambda$ is arational and independent of the choice of sequence $\gamma_{n}$.

The 'unmeasured' lamination $\lambda_{E}$ whose support is the leaves of $[\lambda]$ is called the ending lamination of $E$. If $\xi$ is any other measured lamination whose support is different from that of $\lambda$, then $i(\lambda, \xi)>0$. We can choose the $\gamma_{n}$ to be of bounded hyperbolic length.

Bonahon used the existence of this ending lamination to prove that such an end $E$ is topologically tame. The idea is to construct pleated surfaces $\Sigma_{n}$ in $E$ for which the $\gamma_{n}$ are contained in the bending lamination. Being hyperbolic surfaces, these pleated surfaces have bounded diameter ${ }^{6}$ and also exit the end. They allow one to give $E$ the required product struture $S \times[0,1)$.

Suppose that $M$ is a boundary incompressible hyperbolic 3-manifold. Its end invariants consist of:

(i) The conformal structures on the components of $\Omega / G$ (one for each geometrically finite end) and

(ii) The ending laminations of the geometrically infinite ends.

Theorem 4.66 (Ending lamination theorem $[28,12]$ ). Suppose that $M=\mathbb{H}^{3} / G$ is a boundary incompressible hyperbolic 3-manifold. Then $M$ is uniquely determined up to isometry by its end invariants.

Marden rightly calls this a 'blockbuster theorem'. Expositions of parts can also be found in $[29,27]$. The tameness theorem allows one to get rid of the assumption that $M$ is boundary incompressible. Here is a closely related consequence.

TheOREm 4.67. Suppose that $G_{1}$ and $G_{2}$ are finitely generated and Kleinian groups, and that there is a homeomorphism $\psi: \hat{\mathbb{C}} \rightarrow \hat{\mathbb{C}}$ which induces a type preserving isomorphism $\chi: G_{1} \rightarrow G_{2}$. Suppose also that $\psi$ is conformal on $\Omega\left(G_{1}\right)$. Then $\psi$ is Möbius.

This should be compared to Sullivan's rigidity theorem (see eg [26, 19]) which asserts the same result but only under the much stronger assumption that $\psi$ is quasicomformal.

\footnotetext{
${ }^{6}$ This statement has to be suitably modified if either the $\gamma_{n}$ get very short or if there are cusps.
} 
Manifolds which fibre over the circle Ending laminations are well illustrated with the example of hyperbolic structures on manifolds which fibre over the circle. Start with a hyperbolisable surface $S$, and a pseudo-Anosov map $\phi: S \rightarrow S$. By definition, such a map does not fix any closed curve on the surface. Instead, there is a pair of measured laminations $\lambda^{ \pm}$with the property that $\phi_{*}^{ \pm n}(\gamma) \rightarrow\left[\lambda^{ \pm}\right]$ in $\mathcal{P} \mathcal{M L}$. Thurston showed how to construct a hyperbolic structure on the 3-manifold $M=(S \times[0,1]) / \sim$, where $\sim$ is the equivalence relation which identifies $(x, 1)$ with $(\phi(x), 0)$. This is described in detail in [30]. The cyclic cover $\tilde{M}$ of this manifold has fundamental group $\pi_{1}(S)$ and is a limit of quasifuchsian groups $G\left(\phi_{*}^{n} \omega_{0}, \phi_{*}^{-n} \omega_{0}\right)$ where $\omega_{0}$ is any choice of initial conformal structure on $S$. Thus $\tilde{M}=\mathbb{H}^{3} / G$ for some Kleinian group $G$ isomorphic to $\pi_{1}(S)$. The map $\phi$ induces an isometry $\tilde{M} \rightarrow \tilde{M}$. The manifold $\tilde{M}$ is homeomorphic to $S \times(-\infty, \infty)$ and so has two ends. The geodesic representatives of $\phi_{*}^{n}(\gamma)$ in $\tilde{M}$ exit one end of $\tilde{M}$ and those of $\phi_{*}^{-n}(\gamma)$ the other. Thus the ending laminations of $\tilde{M}$ are exactly the laminations $\left[\lambda^{ \pm}\right]$.

Such groups $G$ provide celebrated examples of Kleinian groups whose limit sets are space filling curves. Involved in the construction of $G$ is the fact that $\Lambda(G)=\hat{\mathbb{C}}$. Let $G_{0}$ be the Fuchsian group such that $\mathbb{H}^{2} / G_{0}$ has the conformal structure $\omega_{0}$. The group $G$ is isomorphic to $G_{0}$. There is a continuous map, called the CannonThurston map, from $S^{1}=\Lambda\left(G_{0}\right)$ to $\Lambda(G)$, providing a continuous mapping from the unit circle $S^{1}$ onto the Riemann sphere $\hat{\mathbb{C}}$. Pictures of such a sequence of limit sets converging to a space filling curve can be found in [31].

\section{REFERENCES}

[1] I. AgOL, Tameness of hyperbolic 3-manifolds, Preprint, GT/0405568, 2005.

[2] A.F. Beardon., The geometry of discrete groups, Springer-Verlag, New York, 1983.

[3] L. Bers, Simultaneous uniformization, Bull. AMS 66 (1960), 94-97.

[4] L. Bers AND I. KrA, A crash course on kleinian groups, Springer Lecture Notes, vol. 400, Springer, New York, 1974.

[5] F. Bonahon, Bouts des variétés de dimension 3, Ann. Math. 124 (1986), no. $1,71-158$. 
[6] M. Bridson And A. Haefliger, Metric spaces of non-positive curvature, Springer Grundlehren, vol. 319, Springer, Berlin, 1999.

[7] J. Brock And K. Bromberg, Cone manifolds and the density conjecture, LMS Lecture Notes, vol. 299, Cambridge University Press, Y. Komori, V. Markovic and C. Series eds., 2003, In Kleinian Groups and hyperbolic 3-manifolds pages 75-94.

[8] J. Brock And K. BromberG, On the density of geometrically finite Kleinian groups, Acta Math. 192 (2004), 33-93.

[9] J. Brock And J. Souto, Algebraic limits of geometrically finite manifolds are tame, to appear in Geom. and Funct. Anal.

[10] D. CAlegari AND D. GABAI, Shrinkwrapping and the taming of hyperbolic 3-manifolds, Preprint, GT/0407161, 2005.

[11] R. CanARY, Ends of hyperbolic 3-manifolds, J. Amer. Math. Soc 6 (1993), 1-35.

[12] R. CANARY J. Brock And Y. Minsky, The classification of Kleinian surface groups II: The ending lamination conjecture, Preprint, GT/0412006, 2005.

[13] R. CAnARY AND S. Hersonsky, Ubiquity of geometric finiteness in boundaries of deformation spaces of hyperbolic 3-manifolds, American J. .Math. 126 (2004), 1193-1220.

[14] M. Culler, Lifting representations to covering groups, Adv. in Math. 59 (1986), 64-70.

[15] D.B.A. Epstein And A. Marden, Convex hulls in hyperbolic space, a theorem of Sullivan, and measured pleated surfaces., LMS Lecture Notes, vol. 111, Cambridge University Press, D.B.A. Epstein ed., 1987, In Analytical and Geometric Aspects of Hyperbolic Space pages 112253. Reprinted in Fundamentals of Hyperbolic Manifolds Selected Expositions, eds. R. D. Canary, A. Marden and D. B. A. Epstein, LMS Lecture Notes 328, 2006.

[16] R. Evans J. Brock, K. Bromberg And J. Souto, Boundaries of deformation spaces and Ahlfors' measure conjecture, Publ. I.H.E.S. 98 (2003), 145-166.

[17] L. Ford, Automorphic functions, Reprint Chelsea, 1972.

[18] S. Hersonsky, R. Canary, M. Culler and P. Shalen, Density of maximal cusps in boundaries of deformation spaces, J. Diff. Geom. 64 (2003), 57-109.

[19] M. KapOvich, Hyperbolic manifolds and discrete groups, Progress in Maths., vol. 183, Birkhäuser, 2001.

[20] P. Laudenbach, A. Fathi and V. Poénaru, Travaux de Thurston sur les surfaces, Astérisque 66-67 (1979), Société Mathématique de France.

[21] C. Maclachlan And A. ReID, The aritheoremetic of hyperbolic 3- 
manifolds, Graduate Texts in Math, vol. 219, Springer-Verlag, 2003.

[22] A. MARDEN, The geometry of finitely generated Kleinian groups, Ann. Math. 99 (1974), 607-639.

[23] A. MARDEn, Outer circles: An introduction to hyperbolic 3-manifolds, Cambridge University Press, 2006.

[24] A. Marden, A proof of the Ahlfors finiteness theorem, LMS Lecture Notes, vol. 329, Cambridge University Press, Y. Komori, V. Markovic and C. Series eds., 2006, In Spaces of Kleinian Groups pages 247-258.

[25] B. Maskit, Kleinian groups, Springer Grundlehren, vol. 287, Springer, Berlin, 1980.

[26] K. Matsuzaki And M. TAniguchi, Hyperbolic manifolds and Kleinian groups, Oxford University Press, 1998.

[27] Y. Minsky, End invariants and the classification of hyperbolic 3manifolds, 2002, Harvard CDM conference, Lecture Notes.

[28] Y. Minsky, The classification of Kleinian surface groups I: models and bounds, Preprint, GT/0302208, 2003.

[29] Y. Minsky, Combinatorial and geometrical aspects of hyperbolic 3manifolds, LMS Lecture Notes, vol. 299, Cambridge University Press, Y. Komori, V. Markovic and C. Series eds., 2003, In Kleinian Groups and hyperbolic 3-manifolds pages 3-40.

[30] J. P. OtaL, Le théorème d'hyperbolisation pour les variétés fibrées de dimension 3, Astérisque 235 (1996), Société Mathématique de France, English Translation: The Hyperbolization Theorem for Fibered 3-Manifolds, SMF/AMS Texts and Monographs 7, 2001.

[31] C. Series D. Mumford And D. Wright, Indra's pearls, Cambridge University Press, 2002.

[32] W. Thurston, Geometry and topology of 3 - manifolds, 1980, Princeton lecture notes, http://www.msri.org/publications/books/gt3m/.

[33] W. Thurston, Three-dimensional geometry and topology, 1997, Princeton lecture notes.

Received February 1, 2006. 